\section{Об основоположниках эндокринной хирургии. Профессор О.В. Николаев и его школа}

\section{С.И. Рыбаков}

ГУ «Институт эндокринологии и обмена веществ им. В.П. Комиссаренко НАМН Украины»
В последние десятилетия наблюдается тенденция присваивать титул «основоположник» той или иной отрасли науки, направления в медицине ученым и практикам, достигшим определенных успехов в этих областях. В частности в хирургии это могут быть впервые выполненные операции при каком-либо заболевании, новый взгляд на патогенез определенной формы патологии и пр. Толкуется это понятие довольно широко и не всегда однозначно. В одном из наиболее авторитетных изданий, 4-томном Толковом словаре русского языка Д.Н. Ушакова, этот термин определяется так: «Основоположник - основатель, создатель какого-нибудь учения, теории, направления, школы». Подобные формулировки содержатся еще в ряде словарей и справочников.

В качестве примера можно обратиться к деятельности некоторых отечественных хирургов, которым принадлежит определенный вклад в развитие эндокринной хирургии и которые считаются основоположниками тех или иных направлений, школ. Профессор Н.А. Ве-

* Адреса для листування (Correspondence): ДУ «Інститут ендокринології та обміну речовин ім. В.П. Комісаренка НАМН України», вул. Вишгородська, 69, м. Київ, 04114 Україна. E-mail: zdovado@ukr.net

(с) С.И. Рыбаков льяминов - выдающийся организатор здравоохранения, военно-полевой, абдоминальный хирург, специалист по заболеваниям костей и суставов, начиная с 1896 г. и примерно до 1910 г. выполнил несколько десятков операций на щитовидной железе. Он высказал ряд соображений по поводу функций щитовидной железы, предложил классификацию ее заболеваний. При выполнении операций он придерживался методик, предложенных Th. Kocher [1-3]. Примерно в это же время известный московский хирург, профессор А.А. Бобров представил аналогичный клинический материал и высказал ряд соображений по поводу оперативного лечения зоба. В частности, он был сторонником энуклеации узлов в противовес рекомендациям Th. Kocher производить эксцизию зоба, не рекомендовал перевязывать тиреоидные артерии на протяжении [3, 4]. Заслуживает внимания монография саратовского профессора В.И. Разумовского «Повреждения и заболевания щитовидной железы» (1903) [5], которая фактически явилась первым руководством по хирургии щитовидной железы в России. В ней был приведен большой объем данных, включающих описания клиники различных форм зоба и опухолей 
Огляди

щитовидной железы, принципы диагностики и лечения, техника операций, меры профилактики и лечения осложнений. Огромная роль в разработке вопросов патологии и хирургического лечения заболеваний щитовидной железы принадлежит профессору А.В. Мартынову $[6,7]$, который от начала XX ст. в течение более 25 лет накопил большой опыт лечения базедовой болезни и других форм зоба, значительно усовершенствовал операцию Микулича, определил показания и противопоказания к этой операции, разработал методики предоперационной подготовки. В его клинике были проведены ценные исследования по гистопатологии, патогенезу базедовой болезни.

На фоне столь внушительной галереи отечественных хирургов, которые внесли большой вклад в изучение различных форм зоба, базедовой болезни, опухолей щитовидной железы, профессора Н.А. Вельяминова считают основоположником эндокринной хирургии в России, тогда как профессорам А.А. Боборову, В.И. Разумовскому, А.В. Мартынову фактически отказано в этом «звании». Можно ли считать основоположниками отечественной эндокринной хирургии этих ученых, если подобные операции на щитовидной железе, хотя и немногочисленные, выполнялись и до них, в том числе великим Н.А. Пироговым? Последний в своих работах по этим вопросам высказывал некоторые теоретические соображения по поводу хирургии зоба и сообщил об одной из первых в России операций под общим обезболиванием [8, 9]. Следует также помнить, что, помимо тиреоидной, у человека существуют другие формы хирургической эндокринной патологии - заболевания надпочечников, паращитовидных, поджелудочной желез, которыми не занимались названные хирурги. Представляется уместным назвать и Н.А. Вельяминова, и А.А. Боброва, и еще названных выше хирургов пионерами тиреоидной хирургии и воздать им должное за смелость и решительность, с которой они вторглись в новую, непознанную область хирургии и достигли определенных обнадеживающих достижений. После них ряд выдающихся отечественных хирургов достигли больших успехов в лечении заболеваний щитовидной железы, предложили новые методики операций, разработали показания и противопоказания для них, принципы предоперационной подготовки, профилактики и лечения осложнений. Другим хирургом, которому отводят роль основоположника эндокринной хирургии, является профессор В.А. Оппель, который на протяжении ряда лет успешно занимался абдоминальной, военно-полевой, сосудистой хирургией, ортопедией и травматологией. В сферу его деятельности также входили вопросы хирургического лечения заболеваний щитовидной, паращитовидных желез, надпочечников, которые были освещены в ряде статей, монографиях «Эндокринологические хирургические наблюдения»» (1926), «Внутренняя секреция» (1929), «Лекции по клинической хирургии и клинической эндокринологии» (1929) и др. Признавая факт, что В.А. Оппель привлек внимание хирургов к эндокринной хирургии в целом, нельзя не отметить, что ряд его концепций и подходов не вполне соответствовали реальности. В частности, ошибочными были его взгляды на роль паращитовидных желез в развитии анкилозирующего полиартрита и предложение удалять несколько желез при этом заболевании. Неверными были его точка зрения на значение надпочечников в происхождении облитерирующего эндартериита (надпочечниковый артериоз) и рекомендации выполнять адреналэктомию при этой патологии. Высказывая ряд современных взглядов на происхождение зоба и тиреотоксикоза, он продолжал оперировать по методике Th. Kocher, был сторонником двухэтапных операций. Тем не менее роль профессора В.А. Оппеля в развитии эндокринной хирургии была чрезвычайно велика. Высказанные им взгляды на характер ряда заболеваний эндокринных желез, их участие в общепатологических процессах в человеческом организме (эндокринная формула), ряд практических рекомендаций для лечения (оригинальный доступ к надпочечнику, переливание крови при тиреотоксикозе и др.) позволяют считать его наиболее крупной фигурой в отечественной эндокринной хирургии 30-х гг. ХХ ст.

Таким образом, краткий экскурс в оценку роли и значения названных хирургов в развитии эндокринной хирургии в нашей стране позволяет предположить, что каждый из них внес определенный вклад в формирование этого направления клинической хирургии, в 
основном тиреоидной. В различные временные периоды их взгляды были основополагающими, им следовали и выполняли их рекомендации. Время вносило коррективы в принципы хирургического лечения эндокринных заболеваний, возникали новые концепции и подходы в соответствии с общим развитием науки. Одновременно появлялись новые фигуры, которые смогли с современных позиций представить обобщенные взгляды на содержание этого направления клинической хирургии, обосновать патофизиологические механизмы развития различных заболеваний эндокринных желез, предложить новые схемы диагностики, новые виды операций, методики обеспечения безопасности и эффективности лечения и реабилитации больных. Ими были воспитаны многочисленные ученики и последователи, которые утвердили и расширили взгляды на патологию не только щитовидной, но и других эндокринных желез.

Фигурой, которая оказалась в состоянии обобщить предшествующие представления о сущности, диагностике и лечении хирургических заболеваний эндокринных желез, совместить их с современными взглядами в единую стройную систему знаний о хирургической эндокринной патологии и предложить новые передовые подходы к их диагностике и лечению, явился профессор Олег Владимирович Николаев (1903-1980), небезосновательно считающийся основоположником современной отечественной эндокринной хирургии.

О.В. Николаев родился 5 марта 1903 г. в Казани, в семье врача. После окончания средней школы в 1919 г. он поступил на медицинский факультет Казанского университета, а затем перевелся в Первый Московский государственный университет, который окончил в 1924 г. Детство и юность О.В. Николаева пришлись на нелегкие времена Первой мировой войны, революции, Гражданской войны, что, возможно, отразилось в последующем на формировании его характера, выработке таких черт, как упорство в достижении цели, способность четко формулировать задачи своей деятельности и добиваться их реализации, способность к трезвому и рациональному мышлению. Все эти качества в сочетании с природными способностями привели к формированию личности талантливого хирурга, исследова- теля, организатора. Еще в студенческие годы он проявил интерес к научной деятельности, основное внимание уделяя анатомии и оперативной хирургии. Результаты проведенного им исследования выявленной сосудистой сети реберных хрящей были опубликованы в отечественном журнале и за рубежом, в Германии. После окончания университета О.В. Николаев работал ординатором кафедры факультетской хирургии Медицинского института, руководимой профессором Н.Н. Бурденко. Здесь он прошел хорошую школу общей хирургии и нейрохирургии. Начиная с 1927 г. О.В. Николаев работал в Институте экспериментальной биологии заведующим лабораторией экспериментальной хирургии. Здесь, под руководством известного ученого Н.К. Кольцова он выполнил ряд работ, касающихся ионной теории возбуждения, в частности роли ионов, гормонов и электролитов в процессах нервного раздражения, влияния гормонов на функцию слюноотделения. В 1931 г. он перешел в Институт эндокринологии Наркомздрава СССР, где возглавил хирургическое отделение, которым руководил до 1974 г. В 1938 г. О.В. Николаев защитил докторскую диссертацию на тему «Материалы к патогенезу и этиологии эндемического зоба», а в 1941 г. был утвержден в звании профессора. Длительность деятельности О.В. Николаева в качестве хирурга-эндокринолога, за вычетом 4 лет войны, составила почти 45 лет. Во время войны он работал доцентом кафедры общей хирургии Киевского медицинского института, который был эвакуирован в Челябинск, и одновременно хирургом эвакуационных госпиталей. В 1943-1945 гг. заведовал отделением Центрального института нейрохирургии, где выполнил более 500 операций по поводу ранений головного и спинного мозга, впервые применил внутриартериальное введение сульфидина при ранениях черепа, по поручению профессора Н.Н. Бурденко руководил клинической группой по испытаниям отечественного пенициллина во фронтовых условиях и в тылу. После 1945 г. деятельность О.В. Николаева неразрывно связана с Московским институтом эндокринологии и химии гормонов. Здесь под его руководством были разработаны современные основы и принципы эндокринной хирургии как самостоятельного направления 
клинической хирургии, эффективные методы лечения многих эндокринных заболеваний, выращена школа высококвалифицированных хирургов-эндокринологов, исследователей и осуществлен огромный объем лечебной работы, позволившей возвратить здоровье многим тысячам больных.

Большинству отечественных эндокринологов, хирургов и представителям других специальностей О.В. Николаев известен как выдающийся хирург, ученый, исследователь, достигший больших успехов в области диагностики и хирургического лечения эндокринных заболеваний. Сравнительно мало кто знает о его колоссальной научно-организационной деятельности, направленной на изучение эндемического зоба в СССР, проведение широкомасштабных мероприятий по выявлению этого заболевания, организации профилактических и лечебных мероприятий. С конца 20 -х гг. в стране развернулась активная научно-практическая деятельность по изучению эндемического зоба, который был достаточно широко распространен в ряде регионов страны. О.В. Николаев принимал самое активное участие в этой работе. По его инициативе, под его руководством и при непосредственном участии организовывались многочисленные экспедиции в районы зобной эндемии в Средней Азии, на Урале, в Забайкалье, на Алтае, на Кавказе, в центральных областях России. Помимо статистических исследований, отражавших распространенность этой патологии, изучались клинические особенности зоба в различных районах, санитарно-гигиенические условия жизни населения, санитарно-химические свойства питьевой воды, содержание йода в почве, источниках водоснабжения, пищевых продуктах, патогистологические характеристики зоба по секционным и хирургическим материалам. На основании собранных данных были сформулированы теории этиологии и патогенеза эндемического зоба и разработаны практические методы противозобной борьбы, в первую очередь с помощью йодной профилактики с использованием йодированной соли. В организационном плане была создана сеть противозобных пунктов, станций и диспансеров, деятельность которых координировалась Центральной зобной комиссией при Министерстве здравоохранения
СССР, которая, в свою очередь, в своей деятельности опиралась на Всесоюзный институт эндокринологии. В результате практического применения разработанных схем йодной профилактики частота эндемического зоба резко снизилась в большинстве районов, а в некоторых он исчез полностью.

Во всей этой деятельности принимал самое активное руководящее и практическое участие О.В. Николаев. Он участвовал в ряде экспедиций по изучению эндемического зоба, совместно с сотрудниками изучал и анализировал поступающие в Институт эндокринологии материалы, на основании которых были выяснены основные характеристики и закономерности развития эндемического зоба и предложены методы его лечения и профилактики. Он также разработал основные принципы и методики хирургического лечения эндемического зоба. Собранный огромный фактический материал послужил основой для его докторской диссертации (1938) и монографии «Эндемический зоб» (1939) [10]. В последующем она выдержала еще два издания в 1949 и 1955 гг. Следует отметить, что при создании этой работы автор использовал 541 отечественную публикацию по вопросам эндемического зоба, 34 из них были его собственными.

Переходя к оценке хирургической деятельности профессора О.В. Николаева, следует сказать, что практически нет ни одной области эндокринной хирургии, где бы он и его ученики не оставили значительный след. В хронологическом плане еще в 30-е гг., занимаясь проблемой эндемического зоба, он уделял большое внимание вопросам хирургического лечения этой патологии. Значительная часть больных с диффузным эндемическим зобом по его рекомендациям нуждались в консервативной терапии микродозами йода, тиреоидином, симптоматическими средствами. Оперативное лечение было показано больным с узловыми формами, но после строгого отбора. Допускалась возможность наблюдения за частью больных с узловым зобом. Случаи диффузного эндемического зоба подлежали операции только при наличии синдромов компрессионного синдрома, нарушений глотания, дыхания. О.В. Николаев часто ссылался на высказывание известного хирурга Recher: «...операция по поводу диффузного зоба явля- 
ется признаком неинтеллигентности хирурга». В вышеназванной монографии лечению эндемического зоба посвящен целый раздел. Были сформулированы показания и противопоказания для операции, предложены оригинальные варианты оперативных вмешательств с учетом распространенности зоба, наличия признаков гипо- или эутиреоза, возраста и состояния больных, детально проанализированы возможные осложнения и способы их профилактики. Следует отметить, что сам О.В. Николаев оперировал большое количество больных с эндемическим зобом как в Институте, так и во время многочисленных экспедиций в разных регионах. В этот период с его участием было оперировано около 1800 больных с узловыми формами зоба без летальных исходов. Была отмечена характерная деталь - по мере успехов внедрения йодной профилактики в районах зобной эндемии сокращалось количество больных, нуждающихся в оперативном лечении.

Начиная со времени, когда О.В. Николаев возглавил хирургическое отделение Института эндокринологии, здесь под его руководством развернулась многоплановая научноисследовательская деятельность и не менее интенсивная хирургическая активность. В клинике изучались вопросы хирургического лечения заболеваний практически всех желез внутренней секреции.

Наиболее выдающимися были успехи О.В. Николаева в лечении тиреотоксического зоба. Это направление в течение многих лет было приоритетным в научно-практической деятельности клиники. Первые работы О.В. Николаева, посвященные хирургическому лечению базедовой болезни, появились в середине 30-х гг. [11, 12]. В них обсуждались показания и противопоказания к операции, ряд деталей техники, исходы лечения. В последующие годы им была разработана новая методика хирургического лечения диффузного токсического зоба - субтотальная, субфасциальная резекция щитовидной железы $[13,14]$. Изучив опыт предшествующих хирургов и самостоятельно оперируя большое количество больных, О.В. Николаев смог предложить новый, безопасный и эффективный тип операции, которая, без преувеличения, стала началом целой эпохи в хирургическом ле- чении тиреотоксикоза. Операция получила практически повсеместное распространение в СССР и ряде стран Восточной Европы. Преимуществами ее при правильном исполнении были высокая эффективность, радикальность и безопасность. Операция выполнялась в пределах четвертой фасции шеи, без перевязки магистральных тиреоидных сосудов на протяжении, что позволяло у подавляющего большинства больных предупредить повреждение возвратных нервов и паращитовидных желез. Сохранение адекватного объема тиреоидной паренхимы путем формирования «культей» обеих долей до 2,0-4,0 г по бокам трахеи позволяло обеспечивать поддержание эутиреоидного состояния и избегать развития гипотиреоза после операции. Параллельно подобный маневр являлся способом предупреждения рецидива заболевания. Важным моментом также явилась разработка методики местного обезболивания, инфильтрационной анестезии 0,25-0,5\% раствором новокаина по методу Вишневского.

Впервые описание методики субтотальной субфасциальной резекции щитовидной железы появилось в 1951 г. в журнале «Хирургия» [13] и в 1952 г. в монографии О.В. Николаева «Хирургия эндокринной системы» [14]. Об этой книге следует сказать особо. Вышедшая в первые послевоенные годы на ломкой, желтой бумаге, без твердого переплета, внешне непрезентабельная, эта работа явилась первой многоплановой отечественной монографией, которая полностью была посвящена хирургической патологии эндокринных желез, начиная от гипофиза и до половых желез. В ней с позиций взглядов того времени детально представлены данные о патогенезе, клинических характеристиках и методах хирургического лечения заболеваний всех эндокринных желез. Один из основных разделов посвящен патологии щитовидной железы: изложены и детально охарактеризованы основные формы тиреоидной патологии, дана классификация зоба и, главное, описаны хирургические методы лечения эндемического и спорадического узлового и диффузного зоба, базедовой болезни, опухолей и других заболеваний (кисты, тиреоидиты и др.). Наибольший интерес представляли результаты применения новой операции при тиреотоксическом зобе. До 1949 г. 
в клинике по поводу базедовой болезни было оперировано 1832 больных. При обследовании в различные сроки после операции установлено, что базедова болезнь была полностью ликвидирована у 98-99\% пациентов, трудоспособность восстановилась у $86 \%$. Всего в клинике к этому времени было оперировано около 3500 больных. Общий процент рецидивов равнялся $0,4 \%$, летальность $-0,3 \%$. К 1955 г. количество оперированных больных превысило 4500, из них 2500 операций были выполнены по поводу тиреотоксического зоба по методике О.В. Николаева [10, 14]. В последующие годы опыт лечения различных форм зоба неуклонно увеличивался. Росло количество операций - к 1967 г. оно достигло 10000 , оттачивались и усовершенствовались детали оперативной техники, повышалось мастерство хирургов. Клиника стала всесоюзным научнометодическим центром лечения различных форм тиреоидной патологии, а предложенная профессором О.В. Николаевым методика субтотальной субфасциальной резекции щитовидной железы стала операцией выбора при тиреотоксическом зобе. Своеобразным промежуточным финишем, подводящим итоги более чем 25-летнего изучения заболеваний щитовидной железы, явилась монография И.Б. Хавина и О.В. Николаева «Болезни щитовидной железы» (1961) [15]. В ней описаны практически все виды тиреоидной патологии. Специальный раздел посвящен хирургическим методам лечения.

С именем профессора О.В. Николаева связано приоритетное систематизированное и углубленное развитие хирургии надпочечных желез в СССР. До него в отечественной литературе появлялись описания единичных случаев опухолей коры надпочечников, практически отсутствовали методы диагностики, а исходы хирургического лечения в большинстве случаев оказывались неблагоприятными. Фактически он стал основоположником этого сложнейшего раздела не только эндокринной хирургии, но и клинической хирургии в целом. Топографо-анатомическое расположение, тяжесть заболеваний надпочечников с многочисленными осложнениями, технические сложности оперативных вмешательств и частые неблагоприятные исходы лечения являлись сдерживающими факторами развития адреналовой хирургии. Основными формами патологии надпочечников являлись опухоли, гормон-продуцирующие и гормонально неактивные, и случаи гиперпластических поражений надпочечников с клиникой болезни Иценко - Кушинга. Вопросами клиники, диагностики и лечения хирургических заболеваний надпочечников О.В. Николаев и его сотрудники начали заниматься с 1934 г. В 1948 г. он сообщил о 7 успешно оперированных больных с опухолью коры надпочечника, описал клинику и предложил классификацию опухолей, остановился на некоторых вопросах лечебной тактики [16]. К 1956 г. количество наблюдений увеличилась до 51 [17]. Описывая эту серию больных, автор подробно остановился на показаниях к операции, вопросах выбора оперативного доступа, улучшении результатов лечения после назначения в послеоперационный период препаратов коры надпочечников [12]. В опубликованной им совместно с Е.И. Таракановым монографии «Гормонально активные опухоли коры надпочечника» (1963) [18] сообщается о 77 оперированных больных. Авторами с передовых позиций того времени подробно описаны морфология, физиология и биохимия коры надпочечников и исходящих из них опухолей, представлена детальная клиническая характеристика различных видов опухолей, изложены методы диагностики, дифференциальной диагностики и основные принципы хирургического лечения. Авторы разделяли опухоли по характеру их гормональной секреции и выделяли новообразования с клиникой глюкокортикоидного, андрогенного, эстрогенного, минералокортикоидного гиперкортицизма. Подобный подход был положен в основу разработанной классификации опухолей коры надпочечников, которая была доложена и вызвала большой интерес на Международном конгрессе эндокринологов в 1960 г. в Копенгагене. Возможности лабораторной диагностики в то время исчерпывались определением 17-ОКС и 17-КС в суточной моче, изредка - кортизола и альдостерона. Методом выбора для визуализации надпочечников были пневморентгенографические исследования, пневоморетроперитонеография и пневморетроперинефрос. Большое внимание было уделено вопросам хирургической тактики и техники при различных видах опухолей 
(выбор доступа, детали хирургической техники и др.). Особое внимание обращено на профилактику и лечение послеоперационной надпочечниковой недостаточности. Это была первая отечественная монография подобного рода и в течение ряда лет являлась руководством, которым пользовались хирурги.

В 1970 г. была опубликована совместная монография О.В. Николаева и В.И. Керцмана «Кортикостерома» [19], в которой сообщалось о 160 оперированных больных, а к 1977 г. клиника Института располагала опытом оперативного лечения 300 больных с гормон-продуцирующими опухолями коры надпочечников [20]. Он был обобщен в докторской диссертации его ученика В.И. Керцмана [21].

Под руководством профессора О.В. Николаева началось развитие метода хирургического лечения болезни Иценко - Кушинга на почве двусторонней гиперплазии коры надпочечных желез. В тот период высказывались немногочисленные мысли о роли гипофиза, точнее базофильной аденомы, в патогенезе этого заболевания. Однако возможности оперативных вмешательств на гипофизе были ограничены, и не всегда удавалось обнаружить аденому. В связи с этим внимание эндокринологов было сосредоточено на попытках подавить функциональную активность коры надпочечников при болезни Иценко - Кушинга. Наиболее реальным путем достижения этих целей являлись операции на надпочечниках. Попытки лечения болезни Иценко - Кушинга путем односторонней адреналэктомии не увенчались успехом. В Институте эндокринологии до 1960 г. было выполнено 16 подобных операций, и ни в одном случае положительных результатов не достигнуто [22]. Попытки расширить объем операции до субтотальной адреналэктомии (удаление до 90\% ткани обоих надпочечников) в большинстве случаев также не увенчались успехом из-за частых рецидивов или развития хронической надпочечниковой недостаточности. В связи с этим хирурги начали постепенно переходить к двусторонней тотальной адреналэктомии. Эта эволюция коснулась и клиники О.В. Николаева, где с 1960 г. стали выполнять подобные операции. К 1963 г. в клинике было оперировано 70 больных, к 1965 г. - 119, из них в 34 случаях проведена тотальная адреналэктомия [22, 23]. В 1966 г. ученик О.В. Николаева А.П. Кали- нин сообщил о 160 операциях, из которых в 75 случаях была проведена тотальная адреналэктомия с летальностью 5,8\% [24]. Была доказана эффективность тотальной адреналэктомии в плане ликвидации симптоматики болезни Иценко - Кушинга. Серьезным сдерживающим моментом являлись сложности лечения и профилактики острого и хронического гипокортицизма, возникающего после тотальной адреналэктомии. Только с получением эффективных препаратов глюкокортикоидных гормонов эта задача была решена.

Одним из интересных и актуальных исследований, проводимых в клинике, было изучение острой и хронической недостаточности коры надпочечников, нередко сопровождавшей вмешательства по поводу опухолей и всегда гиперпластических заболеваний. Были разработаны и внедрены в практику схемы до-, интра- и послеоперационного введения/приема препаратов коры надпочечников (кортин, дезоксикортикостерон, кортизон). Для лечения и профилактики хронической надпочечниковой недостаточности практиковалась подкожная аутотрансплантация фрагментов коры надпочечника больным после тотальной адреналэктомии по поводу болезни Иценко - Кушинга. Подобные операции были выполнены у более чем 100 больных, перенесших тотальную адреналэктомию. Обследование этих больных показало, что аутотрансплантация фрагментов коры надпочечника способствовала более полной коррекции послеоперационного гипокортицизма. Больные после аутотрансплантации чувствовали себя лучше, чем на фоне пероральной заместительной терапии, имелась возможность уменьшать дозы принимаемых глюкокортикоидов. Было доказано, что аутотрансплантаты жизнеспособны, обладают явной секреторной активностью и создают определенный резерв естественных кортикостероидов [24, 25]. Опыт хирургического лечения гиперпластических заболеваний надпочечников отражен в многочисленных публикациях и докторской диссертации ученика О.В. Николаева А.П. Калинина «Хирургическое лечение болезни Иценко Кушинга» (1966) [24]. Во всех этих исследованиях продемонстрирована эволюция клинических, диагностических и хирургических взглядов на опухолевую и гиперпласическую патологию коры надпочечников. 
Огляди

Наряду с опухолями коркового слоя, в клинике широко изучались опухоли, исходящие из мозгового вещества надпочечников - феохромоцитомы. В 1940 г. О.В. Николаев выполнил третью в СССР, после А.Н. Бакулева и С.И. Спасокукоцкого, операцию по поводу феохромоцитомы с благоприятным исходом; эта операция была 110-м случаем из числа описанных в литературе [14]. В 1965 г. О.В. Николаев с соавторами опубликовали монографию «Феохромоцитома» [27]. Как и для опухолей коры надпочечников, в ней детально описаны клинические варианты этого вида новообразований, их морфологические характеристики, методы диагностики и оперативного лечения, предложена классификация. Это также была первая в СССР монография подобного рода. В последующем его ученик К.Н. Казеев в своей докторской диссертации «Доброкачественные и злокачественные опухоли хромаффинной ткани. Хирургическое лечение и отдаленные результаты» (1974) [28] обобщил опыт хирургического лечения 120 больных феохромоцитомой. В работе представлены современные взгляды на патогенез опухолей хромаффинной ткани, описана классификация, клиническая картина, принципы диагностики и лечения хромаффинных опухолей. На тот период данный объем клинических данных являлся одним из крупнейших в мире.

Таким образом, заслугой профессора О.В. Николаева является то, что он привлек внимание клиницистов и начал разработку нового направления эндокринной хирургии лечения заболеваний надпочечников, тогда еще исключительно редкой формы патологии. Им были систематизированы и классифицированы клинические варианты опухолей коры и мозгового вещества надпочечников, разработаны методы гормональной диагностики и визуализации желез и, главное, подробно охарактеризованы принципы хирургического лечения, показания и подготовка к операции, обезболивание, доступы к надпочечникам, детали удаления опухоли, возможные осложнения, методы их профилактики и лечения, ведение послеоперационного периода и отдаленные результаты лечения. Подобные исследования были проведены для гиперпластической патологии коры надпочечников, болезни Иценко - Кушинга. Фактически эти данные можно рассматривать как развитие нового направления в эндокринной хирургии.

В зоне интересов профессора О.В. Николаева находилась также патология паращитовидных желез, которой он начал заниматься с конца 30-х гг. Первая операция по поводу гиперпаратиреоза на почве аденомы паращитовидной железы была проведена им в 1938 г., а к 1952 г. он представил материалы 16 подобных операций [14]. В упоминаемой выше монографии «Хирургия эндокринной системы» он подробно остановился на характеристике разных форм паратиреоидной патологии: гипо- и гиперпаратиреозе, преимущественно на последнем. С позиций знаний того времени описаны патогенез, клинические характеристики, лечебные подходы к этим заболеваниям. О.В. Николаев критически отнесся к взглядам В.А. Оппеля на роль паращитовидных желез в развитии анкилозирующего полиартрита и некоторых других заболеваний и предложениям выполнять при них удаление нескольких паращитовидных желез. В этой первой серии наблюдений большое внимание было уделено методикам операций, в частности поискам паращитовидных желез, особенно в случаях их атипичной локализации. К другому предложению В.А. Оппеля - лечить гипопаратиреоз путем подсадок «бульонных косточек» О.В. Николаев отнесся положительно и не раз применял его в своей практике.

Хотя гиперпаратиреоз считался очень редким заболеванием, тем не менее в клинике проводились интенсивные исследования, посвященные в первую очередь диагностике и тактике хирургического лечения [29]. Результаты этих исследований были представлены в монографии О.В. Николаева и В.Н. Таркаевой «Гиперпаратиреоз» [30], опубликованной в 1974 г. К этому времени в клинике были оперированы 103 больных с гиперпаратиреозом. На фоне современных статистик, насчитывающих многие сотни и даже тысячи наблюдений, эта цифра представляется малозначительной. Однако, учитывая ограниченные возможности диагностики в то время, данный опыт трудно переоценить. Появление этой книги имело еще один положительный момент, т.к. она явилась в определенной степени руководством для хирургов, которые в то время редко сталкивались с этой патологией. 
В монографии содержались подробные сведения об анатомии, морфологии, физиологии паращитовидных желез и их нарушениях при различных формах гиперпаратиреоза первичном, вторичном, третичном, а также их классификация. В ней подробно описаны клинические проявления гиперпаратиреоза, включая костные, почечные, сердечно-сосудистые, психоневрологические синдромы. Отдельно рассмотрены картина гиперпаратиреоидного криза, течение заболевания в детском и юношеском возрасте, злокачественные опухоли паращитовидных желез. Из числа лабораторных показателей основное значение для диагностики придавалось гиперкальциемии, гипофосфатемии, повышению содержания кальция в суточной моче; менее демонстративной считали гипофосфатурию. Многочисленные функциональные пробы - с нагрузкой кортизоном, 5\% хлористым натрием, глюкагоном, тиазидовыми диуретиками и др., обладали сомнительной диагностической ценностью. При костных формах заболевания большое значение отводилось рентгенологическому исследованию костей, а при почечных - внутривенной пиелографии. Топические методы диагностики паратиреоидных опухолей практически отсутствовали.

Не обошел своим вниманием О.В. Николаев такую редкую форму эндокринной хирургической патологии, как гормонально активные опухоли поджелудочной железы из островков Лангерганса - инсуломы (инсулиномы). Исследования этой патологии совместно с ним выполняли Э.Г. Вейнберг, Р.А. Манушарова. Итоги их освещены в серии статей и совместной с Э.Г. Вейнбергом монографии «Инсулома» (1968) [31]. Следует отметить, что описание первых случаев инсуломы содержалось еще в монографии О.В. Николаева [14]. Им в 1951 г. была выполнена вторая в СССР операция по удалению инсулин-продуцирующей аденомы поджелудочной железы; первая была произведена А.Д. Очкиным в 1949 г. В упомянутой монографии содержались исчерпывающие на то время сведения об этом редком заболевании. Авторы выделили две основные формы гиперинсулинизма, протекающего с клиникой гипогликемии: панкреатические, обусловленные заболеваниями поджелудочной железы (аденома, гиперплазия клеток островков Лан- герганса) и внепанкреатические, являющиеся следствием заболеваний печени, эндокринных желез (поражения гипофиза, щитовидной железы, надпочечников), нарушений метаболизма. Подробно описана симптоматика и диагностика инсуломы, гипогликемической болезни. Дана характеристика основных вариантов течения заболевания: галопирующее с частыми приступами гипогликемии, с нарушениями со стороны нервной системы, психики и вариант, когда приступы гипогликемии возникают с большими промежутками (6-12 месяцев) и постепенно учащаются; форма с длительным периодом симптомов-предвестников (повышенный аппетит, чувство голода, головные боли, слабость, прибавка массы тела и др.). Диагностика инсуломы базировалась на анализе клинической картины заболевания и результатов пробы с голоданием. Сущность ее состояла в возникновении симптомов гипогликемии на фоне голодания, снижении уровня глюкозы в крови менее 50-60 мг\%, купировании приступа внутривенным введением 40\% раствора глюкозы или сладкой пищей (триада Wipple). Операции по поводу инсуломы относятся к числу сложных и опасных. Авторы пользовались лапаротомным доступом. Подход к железе осуществлялся через lig. gastrocolicum. Предпочтительной считалась энуклеация опухоли в случае ее обнаружения; подчеркивался риск осложнений в виде послеоперационного панкреатита, повреждения селезеночных сосудов. В случае благоприятного исхода операции наступало полное выздоровление.

Еще одним направлением, получившим развитие в стенах клиники, явилась эндокринная хирургия детского и юношеского возраста. На основании опыта операций у достаточно большого количества больных с различными заболеваниями был осуществлен тщательный анализ клинической картины и ее особенностей при разных формах эндокринной патологии в детском возрасте. В результате были созданы схемы диагностики и разработаны оптимальные варианты оперативных вмешательств, методы профилактики осложнений и реабилитации больных. В качестве примера можно указать на эволюцию подходов к лечению гормонально активных опухолей коры надпочечников. К 1977 г. количество оперированных детей в возрасте 7 месяцев - 16 лет достигло 
81 , т.е. составило $27 \%$ от общего количества пациентов с опухолями надпочечников [21]. Впервые операцию по поводу опухоли надпочечника с клиникой синдрома Кушинга у девочки 13 лет выполнил О.В. Николаев. Самым молодым успешно оперированным больным был мальчик 7 месяцев [18]. Отмечено, что у детей злокачественные опухоли встречаются почти вдвое чаще, чем доброкачественные. Среди злокачественных опухолей одинаково часто встречаются случаи с клиникой синдрома Кушинга и вирильного синдрома, среди доброкачественных преобладали случаи с клиникой синдрома Кушинга. Обращено внимание на более выраженные изменения со стороны наружных половых органов у детей не только с андростеромой, но и с синдромом Кушинга. Из-за нестабильности компенсаторных систем у детей особое внимание обращалось на подготовку операции с учетом профилактики острого гипокортицизма в послеоперационный период: использовались различные схемы введения препаратов глюкокортикоидных гормонов, подсадки под кожу штифтов ДОКА. Чрезвычайно важным являлось тщательное выполнение технических приемов операций, особенно обеспечение гемостаза [18-21]. Опыт лечения болезни Иценко - Кушинга у детей отражен в докторской диссертации Э.Г. Вейнберга [32], монографии М.А. Жуковского с соавт. [33], ряде статей.

Исключительно редким является гиперпаратиреоз в детском возрасте. Из 103 оперированных в клинике больных у 10 возраст не превышал 18 лет (10-18) [30]. Заболевание одинаково часто встречалось у мальчиков и девочек, в отличие от взрослых, где соотношение женщины/мужчины составляло 2,5/1. Костная форма гиперпаратиреоза была выявлена у 8, смешанная - у 2. У 9 пациентов во время операции были обнаружены аденомы (у одного - 2 и у одного -3$)$ и у одного - гиперплазия паращитовидных желез. После удаления аденомы у всех больных наступило выздоровление и улучшение у одного больного после операции по поводу гиперплазии. Подобный опыт сравнительно невелик, но в то время он являлся довольно значительным и представлял несомненный клинический интерес [30]. Относительно операций на щитовидной железе у детей и подростков нет необходимости говорить, что многим больным с базедовой болезнью разработанная О.В. Николаевым методика способствовала сохранению здоровья и жизни с минимальными риском и количеством осложнений. В монографии М.А. Жуковского, О.В. Николаева, С.Б. Пинского «Заболевания щитовидной железы у детей» (1972) [34] содержится подробное описание практически всех форм тиреоидной патологии в детском возрасте, включая вопросы хирургического лечения.

Большое внимание профессор О.В. Николаев уделял еще одной ветви хирургической эндокринологии - онкоэндокринологии. Практически во всех его монографиях начиная с 1952 г. содержатся разделы, посвященные лечению злокачественных опухолей щитовидной, надпочечных, паращитовидных желез. В этих работах приведены подробные сведения о клинике, диагностике и хирургическом лечении злокачественных новообразований. Даны рекомендации по обследованию подобных больных, выбору методик и объемов оперативных вмешательств, использованию дополнительных методов лечения.

Огромной заслугой профессора О.В. Николаева является создание отечественной школы хирургов-эндокринологов, высококвалифицированных специалистов, исследователей. Под его руководством выполнено 10 докторских и 22 кандидатских диссертации. Сам он является автором более 250 научных работ, 10 монографий. Его ученики достигли больших успехов в эндокринной хирургии, выполняли многочисленные научные исследования, успешно оперировали тысячи больных, многие стали руководителями научных и лечебных подразделений. Сюда следует прибавить многие сотни практических врачей, прошедших стажировку в клинике, которые обогатились передовым опытом эндокринной хирургии. Упомянуть всех просто нет возможности. Тем не менее хотелось бы сказать хотя бы несколько слов о его наиболее выдающихся, талантливых учениках.

Ариан Павлович Калинин - талантливый ученый, прекрасный клиницист. Он начинал свою деятельность с изучения вопросов хирургического лечения болезни Иценко - Кушинга, чему посвящена его докторская диссертация, защищенная в 1966 г. В последующем, ра- 
ботая в Московском областном клиническом институте, он создал отделение эндокринной хирургии, со временем превратившееся во Всероссийский центр эндокринной хирургии, воспитал собственную школу хирургов-эндокринологов. Под его руководством получили развитие актуальные проблемы хирургической эндокринологии, хирургии надпочечников, паращитовидных желез, защищены десятки диссертаций, опубликованы сотни работ. Последние несколько десятилетий он являлся признанным главой и лидером в области эндокринной хирургии. Общение с ним доставляло истинное наслаждение - эрудит, полемист, доброжелательный человек, таким он представал перед собеседниками или с трибун многочисленных съездов и конференций.

Константин Николаевич Казеев, почти вся творческая жизнь которого была связана с хирургической клиникой Института эндокринологии. Многие годы он успешно занимался хирургической и научной деятельностью, преимущественно изучением хромаффинных опухолей надпочечников. По этой проблеме он защитил кандидатскую (1966) и докторскую (1974) диссертации и являлся признанным специалистом в этой области эндокринной хирургии, как и во многих других. Талантливый хирург, исследователь, организатор, он пришел на смену своему учителю и многие годы возглавлял хирургическую клинику Института эндокринологии. Внешне серьезный, сдержанный человек, Константин Николаевич отличался добрым, дружеским отношением к своим коллегам и ученикам.

Вилен Исаакович Керцман - один из наиболее талантливых и ярких представителей школы О.В. Николаева. Занимаясь изучением опухолей коры надпочечников, он в 1975 г. защитил докторскую диссертацию по этой тематике. Помимо этого, он был автором двух капитальных монографий, посвященных адренокортикальным опухолям и опубликованных в 1972 г. (совместно с О.В. Николаевым) и 1977 г. По содержанию, насыщенности научно-практическими данными эти работы могут быть признаны классическими в эндокринной хирургии. Волею судеб В.И. Керцман оказался в эмиграции, в Австралии, но и здесь благодаря своему таланту, энергии, знаниям он сумел подтвердить свой хирургический статус и много лет занимался хирургической деятельностью, был отмечен рядом престижных наград нескольких хирургических обществ. Этот список можно значительно расширить и продолжить.

Достаточно активно О.В. Николаев занимался общественной деятельностью. Он был главным редактором всесоюзного журнала «Проблемы эндокринологии и гормонотерапии» (1955-1963 гг.), редактором отдела «Эндокринология» в БМЭ, членом Правления Московского (с 1946 г.) и членом Президиума Всесоюзного (с 1962 г.) общества эндокринологов. Его научно-практическая и организационная деятельность отмечены присвоением звания Заслуженного деятеля науки РСФСР, орденами Ленина, Знаком Почета, медалями.

Возвращаясь к вопросу о первопроходцах и основоположниках, следует напомнить, что многие годы во многих десятках тысяч протоколов операций по поводу тиреотоксического зоба неизменно фигурирует имя профессора О.В. Николаева. Написанные им совместно с сотрудниками монографии об опухолях коры надпочечников до настоящего времени остаются непревзойденными и являются настольными пособиями хирургов-эндокринологов.

\section{Список использованной литературы}

1. Догаткин ЕК. Материалы къ клинике зоба и его оперативному лечениію по даннымъ академической хирургической клиники профессора Н.А. Вельяминова [диссертация]. СПб; 1909. 128 с. (Dogatkin YeK. Materials for the goiter clinic and its operative treatment according to the data of the academic surgical clinic of professor N.A. Velyaminova [dissertation]. St. Petersburg; 1909. 128 р.).

2. Вельяминов НА. Заболевания щитовидной железы и ихъ хирургическое лъчение. СПБ; 1910. 87 с. (Vel'yaminov NA. Diseases of the thyroid gland and their surgical treatment. St. Petersburg; 1910. 87 p.).

3. Седов ВМ, Мирчук КК. К вопросу об истории становления хирургии щитовидной железы в России. Вестник хирургии им. И.И. Грекова. 2014;1:107-10. (Sedov VM, Mirchuk KK. To the question of the history of the formation of thyroid surgery in Russia. Vestnik khirurgii im. I.I. Grekova. 2014;1:107-10).

4. Бобров АА. Зоб и его лечение. В : 1 Съезд российских хирургов. 1900 дек. 28-30; Москва. 1901, с. 55-63. (Bobrov AA. Goiter and its treatment. In: The 1st Congress of Russian surgeons. 1900 Dec 28-30; Moskva. 1901, p. 55-63).

5. Разумовский ВИ. Повреждения и заболевания щитовидной железы. СПб;1903. 58 с. (Razumovskiy VI. Damages and diseases of the thyroid gland. St. Petersburg; 1903. 58 p.).

6. Мартынов AB. Оперативные методы лечения при болезни Basedowa. Результаты. Показания. В: Шервинский ВД, Сахаров ГП, редакторы. Основы эндокринологии; Ленинград. 1929 c. 281-9. (Martynov AB. Surgical treatment of Basedow's disease. Results. Indications. In: Shervinskiy VD, Sakharov GP, editors. Basics of endocrinology; Leningrad. 1929, p. 281-9).

7. Мартынов АВ. Повреждения и болезни щитовидной железы 


\section{Огляди}

В: Гирголав СС, Мартынов ВА, Федоров СП, редакторы. Руководство практической хирургии; Ленинград. 1933;3:543-63. (Martynov AB. Damages and diseases of the Thyroid Gland. In: Girgolav SS, Martynov VA, Fedorov SP, editors. Manual Surgery; Leningrad. 1933; 3: 543-63).

8. Пирогов НИ. Хирургический вопрос об экстирпации щитовидной железы. Дерпт: 1831 с. (Pirogov NI. Surgical issue of extirpation of the thyroid gland. Derpat: 1831 p.).

9. Пирогов НИ. Отчет о путешествии по Кавказу 1847-1849. Вступит. статья, примечания. Михайлова СС, составитель. Москва: Госмедиздат; 1952. 358 с. (Report on the trip to the Caucasus 1847-1849. Introduction article, notes. Mikhailova SS, compiler. Moskva: Gosmedizdat; 1952. 358 p).

10. Николаев ОВ. Эндемический зоб. Москва: Медгиз; 1955. 256 с (Nikolayev OV. Endemic goiter. Moskva: Medgiz; 1955. 256 p.).

11. Николаев ОВ. Хирургическое лечение базедовой болезни как один из методов ее терапии. Вестник эндокринологии. 1935;13:663-75. ((Nikolayev OV. Surgical treatment of Basedow's disease as one of the methods of its therapy. Vestnik endokrinologii. 1935;1-3:663-75).

12. Николаев ОВ. О ближайших результатах лечения базедовой болезни. Проблемы эндокринологии. 1937;4:549-66. (Nikolayev OV. About the immediate results of the treatment of Basedow's disease. Problemy endokrinologii. 1937;4:549-66).

13. Николаев ОВ. К субтотальной резекции щитовидной железы. Хирургия. 1951; 1:37-50. (Nikolayev OV. To subtotal resection of the thyroid gland. Khirurgiya. 1951; 1:37-50).

14. Николаев ОВ. Хирургия эндокринной системы. Москва; 1952. 192 c. (Nikolayev OV. Surgery of endocrine system. Moskva; 1952. 192 p.).

15. Хавин ИБ, Николаев ОВ. Болезни щитовидной железы. Москва: Медгиз; 1961. 252 с. (Khavin IB, Nikolayev OV. Diseases of the thyroid gland. Moskva: Medgiz; 1961. 252 p).

16. Николаев ОВ. Клиника и хирургическое лечение опухолей коры надпочечника. Советская медицина. 1948; 7:22-4. (Nikolayev OV. Clinic and surgical treatment of adrenal cortex tumors. Sovetskaya meditsina. 1948; 7:22-4).

17. Николаев ОВ. К хирургии заболеваний надпочечников Проблемы эндокринологии и гормонотерапии. 1956; 5: 83-9. (Nikolayev OV. To surgery of adrenal gland diseases. Problemy endokrinologii i gormonoterapii. 1956; 5: 83-9).

18. Николаев ОВ, Тараканов ЕИ. Гормонально-активные опухоли коры надпочечника. Москва: Госмедиздат; 1963. 339 с. (Nikolayev OV, Tarakanov YeI. Hormonally active tumors of the adrenal cortex. Moskva: Gosmedizdat; 1963. 339 p.).

19. Николаев ОВ, Керцман ВИ. Кортикостерома. Москва: Медицина; 1970. 262 с. (Nikolayev OV, Kertsman VI. Corticosteroma. Moskva: Meditsina; 1970. 262 p).

20. Керцман ВИ. Клиника и лечение гормонопродуцирующих опухолей коры надпочечника. Ереван: Айстан; 1977. 319 с. (Kertsman VI. Clinic and treatment of hormone-producing tumors of the adrenal cortex. Yerevan: Aystan; 1977. 319 p).

21. Керцман ВИ. Диагностика и хирургия гормонопродуцирующих опухолей коры надпочечника с различными эндокринными синдромами у взрослых и детей [автореферат]. Москва; 1975 40 c. (Kertsman VI. Diagnosis and surgery of hormone-producing tumors of the adrenal cortex with various endocrine syndromes in adults and children [avtoreferat]. Moskva; 1975. 40 p.).

22. Николаев ОВ, Калинин АП. Хирургия надпочечников. Хирургия. 1965; 9:72-8. (Nikolayev OV, Kalinin AP. Adrenal surgery. Khirurgiva. 1965; 9:72-8)

23. Николаев ОВ, Калинин АП. Материалы к хирургическому лечению болезни Иценко - Кушинга. В: Гипофиз - кора надпочечников. Киев; 1964, с. 134-51. (Nikolayev OV, Kalinin AP. Materials for the surgical treatment of Itsenko-Cushing disease. In The pituitary gland - the adrenal cortex. Kiyev; 1964, p. 134-51).

24. Калинин АП. Хирургическое лечение болезни Иценко - Кушинга [автореферат]. Москва; 1966. 24 с. (Kalinin AP. Surgical treatment of Itsenko-Cushing's disease [avtoreferat]. Moskva; 1966. 24 p.).

25. Nikolayev OV, Il'ina OI, Kertsman VI. Long-term results of the treatment of Itsenko-Cushing's disease. Problemy endokrinologii. 1972;3: 3-7.

26. Ильина ОИ. Отдаленные результаты и реабилитация больных после двусторонней тотальной адреналэктомии по поводу болезни Иценко - Кушинга [автореферат]. Москва; 1978. 22 c. (Il'ina OI. Long-term results and rehabilitation of patients after bilateral total adrenalectomy for Itsenko-Cushing's disease [avtoreferat]. Moskva; 1978. 22 p.).

27. Николаев OB, Меньшиков ВВ, Калинин АП. Феохромоцитома Москва; 1965. 233 с. (Nikolayev OV, Men'shikov VV, Kalinin AP. Feokhromotsitoma. Moskva; 1965. 233 p.).

28. Казеев КН. Доброкачественные и злокачественные опухоли хромаффинной ткани. Хирургическое лечение и отдаленные результаты [автореферат]. Москва; 1974. 36 с. (Kazeyev KN. Benign and malignant tumors of chromaffin tissue. Surgical treatment and long-term results [avtoreferat]. Moskva; 1974. 36 p.).

29. Николаев ОВ. Хирургия желез внутренней секреции и ее задачи. Хирургия. 1962; 12: 3-7. (Nikolayev OV. Surgery of endocrine glands and its tasks. Khirurgiya. 1962; 12: 3-7).

30. Николаев ОВ, Таркаева ВН. Гиперпаратиреоз. Москва: Медицина; 1974. 263 с. (Nikolayev OV, Tarkayeva VN Hyperparathyroidism. Moskva: Meditsina; 1974. 263 p.).

31. Николаев ОВ, Вейнберг ЭГ. Инсулома (хирургическое лечение). Москва: Медицина; 1968. 200 с. (Nikolayev OV, Veynberg EG. Insuloma (surgical treatment). Moskva: Meditsina; 1968. 200 p.)

32. Вейнберг ЭГ. Хирургическое лечение болезни Иценко - Кушинга у детей и подростков [автореферат]. Москва; 1974. 42 с. (Veynberg EG. Surgical treatment of Itsenko-Cushing's disease in children and adolescents [avtoreferat]. Moskva; 1974. 42 p.).

33. Жуковский МА, Волкова ТН, Сударев ПВ. Гиперкортицизм у детей. Минск: Беларусь; 1977. 205 с. (Zhukovskiy MA Volkova TN, Sudarev PV. Hypercorticoidism in children. Minsk: Belarus'; 1977. 205 p.)

34. Жуковский МА, Николаев ОВ, Пинский СБ. Заболевания щитовидной железы у детей. Москва: Медицина; 1972. 262 с (Zhukovskiy MA, Nikolayev OV, Pinskiy SB. Diseases of the thyroid gland in children. Moskva: Meditsina; 1972. 262 p.).

(Надійшла до редакції 12.08.2019р.) 